\title{
Real-Time Economic Dispatch by Accommodating Implications of Uncertain Renewable Energy Resources
}

\author{
Mukesh Kumar Shah, Nikhil Gupta, K. R. Niazi, Anil Swarnkar
}

\begin{abstract}
The integration of considerable renewable energy resources in power grids has imposed high variability in the net load demand to be seen by conventional generating stations. The real-time economic dispatch (RTED) of modern power systems must consider minute-to-minute variability in the net load demand on the station during a scheduling interval of 5-15 min. The existing methods therefore may not explicitly handle economic implications of power system. This paper proposes a new method for RTED while fully addressing variability in power generation from renewable energy resources and load demand. The method suggests mean PFs for committed generators by conducting (off-line) economic dispatch for each subinterval of 1 min. Mean PFs are evaluated only once, that is just at the beginning of scheduling interval and will continued throughout the interval. This reduces complexity and dimensionality of the proposed method. The simulation results on a standard test bench validate economic competence of proposed approach over that conventional one.
\end{abstract}

Index Terms- Real-time economic dispatch, mean participation factor, scheduling interval, renewable energy resources

\section{INTRODUCTION}

The economic operation of power system has assumed greater significance in the context of modern power systems. The competitive business environment, scarcity of energy generation resources, depleting fossil fuel reserve, environmental concerns and exponentially growing energy demand have led to the development and penetration renewable energy resources (RESs) in power systems. fossil fuel based thermal power plants but has made the economic dispatch problem even more important and complex due to minute to minute variability in power generation from these resources.

The purpose of economic dispatch is to allocate generation schedule to various generators to meet the load demand in the most economical way. A lot of research work has been carried out in the area of generation dispatch. Broadly, the

Revised Version Manuscript Received on 10 September, 2019.

Mukesh Kumar Shah, Department of Electrical Engineering, Malaviya National Institute of Technology, Jaipur, India.

(Email: mkshah.ee@mnit.ac.in)

Nikhil Gupta, Department of Electrical Engineering, Malaviya National Institute of Technology, Jaipur, India.

(Email: nikhilgupta.ee@mnit.ac.in)

K. R. Niazi, Department of Electrical Engineering, Malaviya National Institute of Technology, Jaipur, India.

(Email: krniazi.ee@mnit.ac.in)

Anil Swarnkar, Department of Electrical Engineering, Malaviya National Institute of Technology, Jaipur, India.

(Email: aswarnkar.ee@mnit.ac.in) Penetration of RESs has definitely reduced the burden of

economic dispatch (ED) problem can be classified in four different categories namely optimal power flow, ED in relation to automatic generation control (AGC), dynamic economic dispatch (DED) and economic dispatch with non-conventional generation sources [1]. In optimal power flow (OPF) method load flow techniques are used to determine the ED of generators. This method is used for static optimization. In AGC, ED and load frequency control (LFC) are combined. Even though ED and LFC have different time horizons, they are not independent as ED provides the set point for LFC. Now both of these control actions fall under a single activity called AGC [1]. The AGC is successful and provides economic solution if load and frequency changes are small. However, this is not possible when there is large variability in generation. In DED, the cost of generation change (ramp) is taken into consideration unlike static optimization. The DED method uses forecasts of system load to develop optimal generator output trajectories for the next 24 hours. The day-ahead dynamic economic dispatch (DDED) determines the optimal settings of generator units with predicted load demand over the scheduling period. The day-ahead optimal power flow (DA-OPF) under vertically integrated power system is a tool to schedule online generators in order to meet the forecasted demand and reserve requirements at least cost [2]. A hybrid methodology for solving DED is deployed in [3]. A fuzzy-optimization approach to DED, considering the uncertainties in deregulated energy and reserve markets is presented in [4]. The DED works well with accurate load and generation forecast. DED with direct interface with on-line function such as AGC is called on-line economic dispatch or real-time economic dispatch (RTED). A Newton Raphson economic method for solving RTED using Lagrangian augmented function is proposed in [5]. A RTED algorithm suitable for on-line generation control and for study programs is presented in [6]. A high-speed RTED method is proposed in [7]. The method uses the short-term load forecast, which is suitable for power systems with large renewable energy penetration. Conventional DA-OPF and RT-OPF approaches ignore the impact of renewable power generation variability during the scheduling interval [2]. Conventionally all the approaches for RTED, uses scheduling interval of 10-15 min where ED is performed at every scheduling interval. During the period between two consecutive scheduling intervals, the 
generators participate in managing power imbalance, based on participation factors (PFs) from previous ED [8]. The same is repeated for the next scheduling interval. In modern power systems this approach of calculating PFs at the beginning of the scheduling interval may not be very economical owing to the high variability in generations from RESs. In this paper a modified PFs-based approach is proposed for RTED of modern power systems.

\section{PROPOSED MEAN PF METHOD FOR RTED}

The conventional real time economic dispatch is carried for the scheduling interval $T$ while determining PF of generating units from their economic dispatch carried just before the beginning of this interval (base-point generation). These PFs remain unaltered during the scheduling interval. The method was successfully adapted owing to limited variation in minute-to minute variations in load demand that practically not hampers economy of power generation so long as the scheduling interval is varied from 10-15 min.

The integration of renewable energy resources have introduces additional variability and intermittency in power generation. This has imposed serious limitation on real-time economic operation of power systems using conventional PF approach. It happened because the variations in net load demand seen by the generating system are now with measurable variations. Therefore, the PF of generators obtained from the economic dispatch at the beginning of scheduling no more remain so economic for the forthcoming subintervals of the same interval. As a remedy, the economic dispatch may be determined for each subinterval " $t$ " of $1 \mathrm{~min}$. But this is not a practical approach for RTED [8], the PFs of generators must remain same throughout the scheduling interval.

In proposed methodology, the economic dispatch (off-line) is determined for each subinterval " $t$ " of $1 \mathrm{~min}$. considering variability and uncertainty in load demand and power generation from renewables. The PFs of each of these economic generating schedules are then determined. The mean PF of each of the generator is determined. RTED is now determined keeping mean PFs fixed throughout the scheduling interval $T$. The economic schedule for other scheduling intervals of the given scheduling period can also be determined using the same approach. The proposed approach is highly simplified and easy to implement, though it requires repeated application of optimal economic dispatch during the scheduling interval. A fast optimization technique is therefore essential to implement proposed method.

\section{PROBLEM FORMULATION}

For $N_{G}$ committed thermal generating units, $N_{P V}$ solar PVs, $N_{W T}$ WTs and total $N_{s u b}$ subintervals in the scheduling interval $T$, the RTED problem is formulated as follows:

$$
\begin{aligned}
& \text { Minimize } \sum_{t=1}^{N_{\text {sub }}} \sum_{i=1}^{N_{G}} C_{G i}(t) \\
& \text { Where, } C_{G i}(t)=a+b P_{G i}(t)+c P_{G i}^{2}(t) \\
& P_{G i}(t)=P F_{i}^{T} \times P_{G i}(0)
\end{aligned}
$$

Where, $P_{G i}(0)$ is the base point generation, $P F_{i}^{T}$ is the proposed $\mathrm{PF}$ for the $i$ th thermal generator being kept constant during the scheduling interval $T$ and is given by

$$
P F_{i}^{T}=\frac{1}{N_{\text {sub }}} \sum_{t=1}^{N_{s u b}} \frac{P_{G i}^{*}(t)}{\sum_{i=1}^{N_{G}} P_{G i}^{*}(t)} ; P F_{i}(t)=\frac{P_{G i}^{*}(t)}{\sum_{i=1}^{N_{G}} P_{G i}^{*}(t)}
$$

Where, $P F_{i}(t)$ is the PF for the $i$ th thermal generator for the economic dispatch being conducted for the $t$ th subinterval and $P_{G i}^{*}(t)$ is the corresponding economic generation.

Subject to the following constraints

$$
\begin{aligned}
& \sum_{i=1}^{N_{G}} P_{G i}(t)=P_{D}(t)-\sum_{j=1}^{N_{P V}} P_{G j}^{P V}(t)-\sum_{k=1}^{N_{W T}} P_{G k}^{W T}(t) \\
& P_{G i}^{\min } \leq P_{G i}(t) \leq P_{G i}^{\max }
\end{aligned}
$$

Eqn.(5) and (6) represent power balance and generator power limit constraints, respectively. For simplicity, the generator operational constraints viz. prohibited operating zones, ramp rate limits, valve-point loading, etc. are not considered and line losses are ignored in the formulation, but can be easily accommodated.

\section{SIMULATION RESULTS}

The simulations are carried on IEEE 30-bus system [9] having six generators being located at bus 1, 2, 5, 8, 11 and 13. The cost coefficients of generators are taken as shown in Table I. This system is modified by assuming one WT and SPV unit, each of $30 \mathrm{MWp}$ rating, at bus 11 and 13, respectively. The forecasted power generation from these units with one minute time resolution is taken as shown in Fig. 1. The scheduling period " $P$ " is taken of $60 \mathrm{~min}$ which is uniformly divided into six intervals each of 10 min duration. Each interval " $T$ " is composed of ten subintervals " $t$ " each of 1 min duration. The line losses are neglected for simplicity. For the given interval $T$ being extended from 12:01 hr. to $12: 10 \mathrm{hr}$., the forecasted load demand for each subinterval is taken as presented in Table II. The table also shows net demand as seen by the generators which is obtained by subtracting the sum of forecasted power generation from SPV and WT units.

Table I Cost coefficients for six generator test system

\begin{tabular}{|c|c|c|c|}
\hline Generator & $\begin{array}{c}\boldsymbol{a} \\
\mathbf{U S} \mathbf{\text { hr }})\end{array}$ & $\begin{array}{c}\boldsymbol{b} \mathbf{( M W} \\
\mathbf{U S} \mathbf{\$} / \mathbf{h r})\end{array}$ & $\begin{array}{c}\boldsymbol{c}(\mathbf{M W} \\
\mathbf{U S} \mathbf{\mathbf { h }} \mathbf{\mathbf { 2 }})\end{array}$ \\
\hline G1 & 0 & 1.75 & 0.0175 \\
\hline G2 & 0 & 1 & 0.0625 \\
\hline G3 & 0 & 3.25 & 0.00834 \\
\hline G4 & 0 & 3 & 0.025 \\
\hline G5 & 0 & 3 & 0.025 \\
\hline G6 & 0 & 2 & 0.00375 \\
\hline
\end{tabular}

According to proposed method, the off-line economic dispatch is conducted for each subinterval for the net load demand shown in Table II persists during the interval $T$. The results obtained are presented in Table III. From this dispatch, the PF of generators are evaluated for each 
subinterval as shown in Table IV. The table also shows mean $\mathrm{PF}$ of each generator. With these mean PFs remains same during the interval $T$, the economic dispatch obtained for each subinterval is presented in Table V. For this economic dispatch, the fuel cost of each generator is obtained for each

Table II Forecasted load demand and power generation from renewables

\begin{tabular}{|c|c|c|c|c|c|c|c|c|c|c|c|}
\hline $\mathbf{X}$ & 12:00 & 12:01 & 12:02 & 12:03 & 12:04 & $12: 05$ & 12:06 & 12:07 & $12: 08$ & 12:09 & 12.10 \\
\hline $\mathrm{Y}$ & 283.40 & 286.23 & 289.07 & 291.90 & 294.74 & 297.57 & 300.41 & 303.25 & 306.09 & 308.93 & 311.77 \\
\hline $\mathrm{Z}$ & 244.96 & 247.93 & 256.40 & 257.62 & 253.56 & 254.84 & 273.80 & 267.79 & 264.44 & 265.89 & 288.52 \\
\hline
\end{tabular}

X: Time (hr.), Y: Load demand (MW), Z: Net load demand (MW)

Table III Economic dispatch (off-line) for each subinterval

\begin{tabular}{|c|c|c|c|c|c|c|c|c|c|c|c|}
\hline \multirow[t]{2}{*}{ Gen. } & \multicolumn{11}{|c|}{ Time (hrs.) } \\
\hline & 12:00 & 12:01 & 12:02 & 12:03 & 12:04 & 12:05 & 12:06 & 12:07 & 12:08 & 12:09 & 12.10 \\
\hline G1 & 40.408 & 40.907 & 42.333 & 42.537 & 41.854 & 42.070 & 45.258 & 44.247 & 43.685 & 43.929 & 47.705 \\
\hline $\mathrm{G} 2$ & 17.314 & 17.454 & 17.853 & 17.910 & 17.719 & 17.780 & 18.672 & 18.389 & 18.232 & 18.300 & 19.357 \\
\hline G3 & 10.000 & 10.000 & 10.000 & 10.000 & 10.000 & 10.000 & 10.000 & 10.000 & 10.000 & 10.000 & 10.172 \\
\hline G4 & 10.000 & 10.000 & 10.000 & 10.000 & 10.000 & 10.000 & 10.000 & 10.000 & 10.000 & 10.000 & 10.000 \\
\hline G5 & 12.000 & 12.000 & 12.000 & 12.000 & 12.000 & 12.000 & 12.000 & 12.000 & 12.000 & 12.000 & 12.000 \\
\hline $\begin{array}{c}\text { G6 } \\
\text { Gen. }\end{array}$ & $\begin{array}{c}155.23 \\
9\end{array}$ & $\begin{array}{c}157.56 \\
6\end{array}$ & $\begin{array}{c}164.21 \\
8\end{array}$ & $\begin{array}{c}165.17 \\
3\end{array}$ & $\begin{array}{c}161.98 \\
7\end{array}$ & $\begin{array}{c}162.99 \\
4\end{array}$ & $\begin{array}{c}177.87 \\
0\end{array}$ & $\begin{array}{c}173.15 \\
4\end{array}$ & $\begin{array}{c}170.52 \\
8\end{array}$ & $\begin{array}{c}171.66 \\
9\end{array}$ & $\begin{array}{c}189.28 \\
8\end{array}$ \\
\hline Total & $\begin{array}{c}244.96 \\
1\end{array}$ & $\begin{array}{c}247.92 \\
7\end{array}$ & $\begin{array}{c}256.40 \\
4 \\
\end{array}$ & $\begin{array}{c}257.62 \\
1\end{array}$ & $\begin{array}{c}253.56 \\
1 \\
\end{array}$ & $\begin{array}{c}254.84 \\
4 \\
\end{array}$ & $\begin{array}{c}273.80 \\
0\end{array}$ & $\begin{array}{c}267.79 \\
0\end{array}$ & $\begin{array}{c}264.44 \\
4\end{array}$ & $\begin{array}{c}265.89 \\
8\end{array}$ & $\begin{array}{c}288.52 \\
2\end{array}$ \\
\hline
\end{tabular}

Table IV PFs obtained for economic dispatch (off-line)

\begin{tabular}{|c|c|c|c|c|c|c|c|c|c|c|c|c|}
\hline \multirow[t]{2}{*}{ Gen. } & \multicolumn{12}{|c|}{ Time (hrs.) } \\
\hline & $12: 00$ & $12: 01$ & $12: 02$ & $12: 03$ & $12 ; 04$ & $12: 05$ & $12: 06$ & $12: 07$ & $12: 08$ & $12: 09$ & $12: 10$ & Mean PF \\
\hline G1 & 0.165 & 0.165 & 0.165 & 0.165 & 0.165 & 0.165 & 0.165 & 0.165 & 0.165 & 0.165 & 0.165 & 0.1651 \\
\hline $\mathrm{G} 2$ & 0.071 & 0.070 & 0.070 & 0.070 & 0.070 & 0.070 & 0.068 & 0.069 & 0.069 & 0.069 & 0.067 & 0.0692 \\
\hline G3 & 0.041 & 0.040 & 0.039 & 0.039 & 0.039 & 0.039 & 0.037 & 0.037 & 0.038 & 0.038 & 0.035 & 0.0384 \\
\hline G4 & 0.041 & 0.040 & 0.039 & 0.039 & 0.039 & 0.039 & 0.037 & 0.037 & 0.038 & 0.038 & 0.035 & 0.0383 \\
\hline G5 & 0.049 & 0.048 & 0.047 & 0.047 & 0.047 & 0.047 & 0.044 & 0.045 & 0.045 & 0.045 & 0.042 & 0.0460 \\
\hline G6 & 0.634 & 0.636 & 0.640 & 0.641 & 0.639 & 0.640 & 0.650 & 0.647 & 0.645 & 0.646 & 0.656 & 0.6429 \\
\hline
\end{tabular}

Table V RTED using proposed mean PF approach

\begin{tabular}{|c|c|c|c|c|c|c|c|c|c|c|c|}
\hline \multirow[t]{2}{*}{ Gen. } & \multicolumn{11}{|c|}{ Time (hrs.) } \\
\hline & $12: 00$ & $12: 01$ & $12: 02$ & $12: 03$ & $12 ; 04$ & $12: 05$ & $12: 06$ & $12: 07$ & $12: 08$ & $12: 09$ & $12: 10$ \\
\hline G1 & 40.408 & 40.971 & 42.579 & 42.810 & 42.040 & 42.283 & 45.217 & 44.224 & 43.671 & 43.912 & 47.648 \\
\hline $\mathrm{G} 2$ & 17.314 & 17.555 & 18.245 & 18.344 & 18.013 & 18.118 & 18.957 & 18.541 & 18.309 & 18.410 & 19.976 \\
\hline G3 & 10.000 & 10.000 & 10.000 & 10.000 & 10.000 & 10.000 & 10.509 & 10.278 & 10.150 & 10.206 & 11.074 \\
\hline G4 & 10.000 & 10.000 & 10.000 & 10.000 & 10.000 & 10.000 & 10.494 & 10.264 & 10.135 & 10.191 & 11.058 \\
\hline G5 & 12.000 & 12.000 & 12.000 & 12.000 & 12.000 & 12.000 & 12.593 & 12.316 & 12.163 & 12.229 & 13.270 \\
\hline G6 & 155.239 & 157.401 & 163.580 & 164.467 & 161.507 & 162.443 & 176.031 & 172.167 & 170.015 & 170.950 & 185.496 \\
\hline Total & 244.961 & 247.927 & 256.404 & 257.621 & 253.561 & 254.844 & 273.800 & 267.790 & 264.444 & 265.898 & 288.522 \\
\hline
\end{tabular}


Table VI RTED using conventional PF approach

\begin{tabular}{|c|c|c|c|c|c|c|c|c|c|c|c|}
\hline \multirow{2}{*}{ Gen. } & \multicolumn{10}{|c|}{ Time (hrs.) } \\
\cline { 2 - 13 } & $12: 00$ & $12: 01$ & $12: 02$ & $12: 03$ & $12 ; 04$ & $12: 05$ & $12: 06$ & $12: 07$ & $12: 08$ & $12: 09$ & $12: 10$ \\
\hline G1 & 40.408 & 40.897 & 42.296 & 42.497 & 41.827 & 42.038 & 45.165 & 44.174 & 43.622 & 43.862 & 47.594 \\
\hline G2 & 17.314 & 17.524 & 18.123 & 18.209 & 17.922 & 18.013 & 19.353 & 18.928 & 18.691 & 18.794 & 20.393 \\
\hline G3 & 10.000 & 10.121 & 10.467 & 10.517 & 10.351 & 10.403 & 11.177 & 10.932 & 10.795 & 10.855 & 11.778 \\
\hline G4 & 10.000 & 10.121 & 10.467 & 10.517 & 10.351 & 10.403 & 11.177 & 10.932 & 10.795 & 10.855 & 11.778 \\
\hline G5 & 12.000 & 12.145 & 12.561 & 12.620 & 12.421 & 12.484 & 13.413 & 13.118 & 12.954 & 13.026 & 14.134 \\
\hline G6 & 155.239 & 157.118 & 162.490 & 163.261 & 160.689 & 161.502 & 173.515 & 169.706 & 167.585 & 168.507 & 182.844 \\
\hline Total & 244.961 & 247.927 & 256.404 & 257.621 & 253.561 & 254.844 & 273.800 & 267.790 & 264.444 & 265.898 & 288.522 \\
\hline
\end{tabular}

subinterval as shown in Table VI. The total fuel cost for this economic dispatch is found to be US\$ 127.4301 for the interval of $10 \mathrm{~min}$. It is noteworthy that the fuel cost for the minute-to-minute economic dispatch shown in Table III. This implies that the economic dispatch using proposed method costs just $0.016 \%$ higher than that can ever be obtained. Next, the conventional PF approach suggests PF as shown in Table

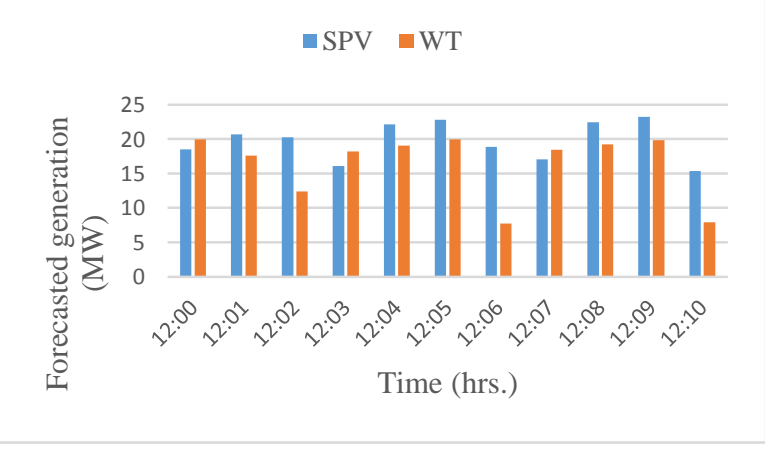

(a)

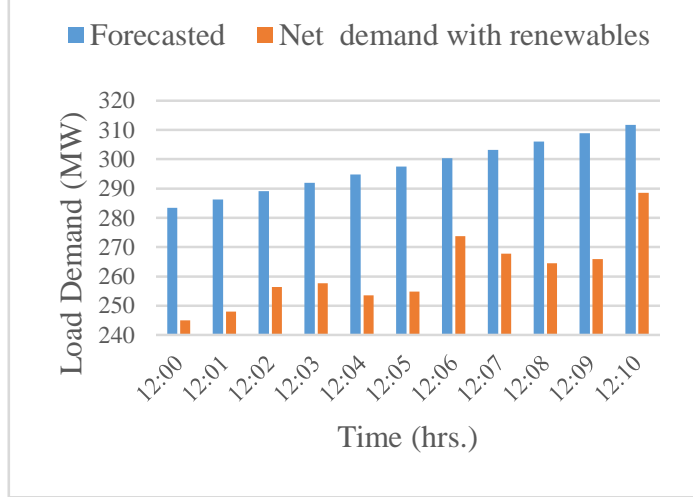

(b)

Fig. 1 (a) Power generation from solar PV and WT (b) forecasted load demand and net demand during a scheduling period

IV for the base-point generation, i.e. at $12.00 \mathrm{hrs}$. According to this approach if these PFs remain unaltered during the interval $T$, the economic dispatch will be as shown in Table VI. For this economic dispatch the total fuel cost is found to be US\$ 127.506 which is $0.060 \%$ higher than the economic dispatch if conducted for each subinterval. This shows that conventional PF method may not be optimal for economic dispatch in the presence of renewable energy interval is found to be US\$127.4095 while evaluated for the resources.

\section{DISCUSSION}

The proposed mean PF method enables better economic dispatch which is produces results as if economic dispatch is obtained for each subinterval. The mean PFs derived obtained by considering variability and intermittency in the power generation from renewables and load demand pertaining to each subinterval. The mean PFs thus captures aggregate net load demand of the system whereas conventional PF approach is based upon net load demand on the system just before the beginning of an interval. Since the integration of renewables enhanced variability in the net load demand, the conventional PF approach that acts well in the absence of renewables no more remains accurate for the economic dispatch while considering SPVs and WTs.

\section{CONCLUSION}

This paper proposes mean PF approach for RTED of thermal units in the presence of SPV and WT units. Proposed method off-line conducts economic dispatch for each subinterval of $1 \mathrm{~min}$ and employs the mean of PFs for each of these economic dispatches that remain unchanged throughout the interval $T$. The simulation results on a standard test bench shows that the fuel cost during the interval using the proposed method is very much economic. It has also been observed that existing PF approach for RTED becomes uneconomic when renewables being integrated in the system. The proposed method is highly simplified and promising to handle uncertainty and variability in load demand and power generation from renewables. The generator operational constraints such as prohibited operating zones, valve-point loading, ramp rate limits and variability cost involved in the objective function are ignored for simplicity, but can also be considered for more practical economic dispatch solutions.

\section{REFERENCES}

1. B. H. Chowdhury and S. Rahman, "A review of recent advances in economic dispatch," in IEEE Transactions on Power Systems, vol. 5, no. 4, pp. 1248-1259, Nov. 1990.

2. S. S. Reddy and P.R. Bijwe, "Day-Ahead and Real Time Optimal Power Flow considering Renewable Energy Resources," in International Journal of Electrical Power \& Energy Systems, vol. 82, pp. 400-408, Nov. 2016. 
3. A P. Attaviriyanupap, H. Kita, E. Tanaka and J. Hasegawa, "A hybrid EP and SQP for dynamic economic dispatch with non-smooth fuel cost function," in IEEE Transactions on Power Systems, vol. 17, no. 2, pp. 411-416, May 2002.

4. P. Attaviriyanupap, H. Kita, E. Tanaka and J. Hasegawa, "A fuzzy-optimization approach to dynamic economic dispatch considering uncertainties," in IEEE Transactions on Power Systems, vol. 19, no. 3, pp. 1299-1307, Aug. 2004.

5. Y. C. Chang, W. T. Yang, C. C. Liu, "On-line economic dispatch using a new loss coefficient formula," in Electric Power Systems Research, vol. 25, no. 2, pp. 131-136, Nov. 1992.

6. G. B. Sheble, "Real-time economic dispatch and reserve allocation using merit order loading and linear programming rules," in IEEE Transactions on Power Systems, vol. 4, no. 4, pp. 1414-1420, Nov. 1989.

7. N. Yorino, H. M. Hafiz, Y. Sasaki and Y. Zoka, "High-Speed Real-Time Dynamic Economic Load Dispatch," in IEEE Transactions on Power Systems, vol. 27, no. 2, pp. 621-630, May 2012.

8. S. Surender Reddy, P. R. Bijwe and A. R. Abhyankar, "Real-Time Economic Dispatch Considering Renewable Power Generation Variability and Uncertainty Over Scheduling Period," in IEEE Systems Journal, vol. 9, no. 4, pp. 1440-1451, Dec. 2015.

9. [Online].Available:http://www.ee.washington.edu/resear ch/pstca 